Barbara Flückiger* und Anna Bohn

\title{
Digitalisierung und filmwissenschaftliche Forschung
}

\author{
Prof. Dr. Barbara Flückiger im Interview mit Dr. Anna Bohn
}

https://doi.org/10.1515/bfp-2020-2049

Zusammenfassung: Mit der Digitalisierung des Films eröffnen sich neue Methoden und Forschungsfelder, denn die Wahrnehmung von Filmgeschichte hat sich durch diesen Umbruch nachhaltig verändert. Video-Streaming ist dabei eingebettet in einen Knotenpunkt zwischen Zugang zu Filmwerken, aber auch neuen Formen der Rezeption und Filmgeschichtsschreibung in Forschung und Lehre. Methoden und Werkzeuge der Digital Humanities sind ein vielversprechendes Feld für die Erforschung von Filmen, ihrer Geschichte, der Ästhetik und Narration. Entwicklungen in den Forschungsprojekten zu Filmfarben am Seminar für Filmwissenschaft der Universität Zürich zeigen exemplarisch auf, welche Potenziale mit den neuen Technologien und Ansätzen verbunden sind.

Schlüsselwörter: Filmwissenschaft; Digital Humanities; Filmästhetik; Materialität; Restaurierung; Digitalisierung; Zugang; Metadaten

\section{Digitalisation and Research in Film Studies}

Abstract: The digitization of film opens up new methods and fields of research, because the perception of film history has changed permanently as a result of this transition. Video streaming is changing access to films but enables also new forms of reception and film historiography in research and teaching. Digital humanities methods and tools are a promising field for researching films, their history, aesthetics, and narration. In the framework of research projects on film colors at the Department of Film Studies at the University of Zurich a variety of such developments have been developed that are exemplary for the potential associated with the new technologies and approaches.

Keywords: Film studies; digital humanities; film aesthetics; materiality; restoration; digitization; access; metadata

*Kontaktperson: Prof. Dr. Barbara Flückiger, baflueckiger@gmail.com

Dr.Anna Bohn, anna.bohn@zlb.de

\section{Interview mit Prof. Dr. Barbara Flückiger}

Seminar für Filmwissenschaft der Universität Zürich, Projektleiterin und Principal Investigator des Projekts ERC Advanced Grant FilmColors. Bridging the Gap Between Technology and Aesthetics

Die Fragen stellte Dr. Anna Bohn, wissenschaftliche Referentin strategische Filmentwicklung, Zentral- und Landesbibliothek Berlin.

Anna Bohn (AB): Zu Deinen Forschungsschwerpunkten in der Filmwissenschaft zählen die Digitalisierung des Films, die Interaktion zwischen Technologie und Ästhetik und auch Innovationstheorie. Wie verändert der Online-Zugang zu Filmen und die damit verbundene Technologie die Ästhetik und Wahrnehmung des Films?

Barbara Flückiger (BF): Der Online-Zugang ist eine wesentliche Facette des Umbruchs von analogen zu digitalen Formaten. Mit der Digitalisierung des Films hat sich die Wahrnehmung massiv verändert, wir erforschten die Frage, wie digitale Techniken die Rezeption und Zirkulation von Filmgeschichte verändern im Projekt Filmgeschichte Re-Mastered. Memopolitische, ästhetische und wahrnehmungstheoretische Konsequenzen der digitalen Medientransition, ${ }^{2}$ zusammen mit Franziska Heller.

Man muss dabei verschiedene Ebenen berücksichtigen. Materielle, ästhetische, technische, epistemologische und die symbolische Ebene, wie Digitalität als Praxis durch Diskurse aufgeladen wird.

Technisch und materiell gibt es keine Kontinuität zwischen analogen und digitalen Filmelementen, es sind fundamental andere Methoden der Enkodierung und damit auch der Erscheinungsweise. So habe ich in meiner Habili-

1 https://www.film.uzh.ch/de/research/projects/verbund/ercfilmco lors.html.

2 https://www.film.uzh.ch/de/research/projects/archive-projects/ar chive/remastered.html. 


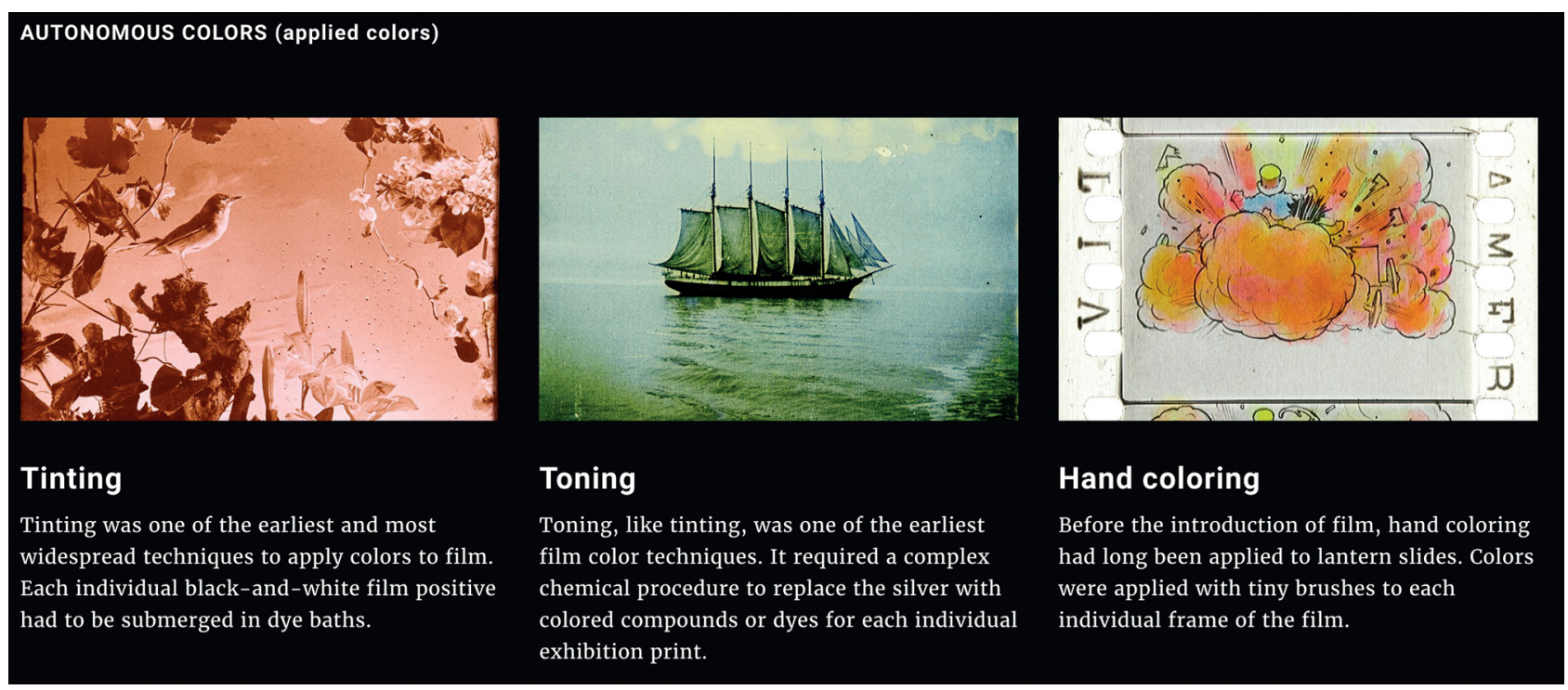

Abb. 1: Timeline of Historical Film Colors, Screenshot Startseite

tationsschrift Visual Effects. Filmbilder aus dem Computer ${ }^{3}$ beschrieben, dass digitale Elemente opak und polymorph sind. Wir haben über die Wahrnehmung keinen direkten Zugang zu digitalen Informationen, sie sind abstrakt durch einen mathematischen Code definiert. Durch diese abstrakte Enkodierung sind die Informationen aber auch polymorph, sie sind nicht an einen bestimmten Träger gebunden wie analoger Film, sondern man kann diese Informationen in Stein meißeln, auf verschiedenen Datenträgern sichern oder auch gentechnisch durch DNA repräsentieren.

Durch diese mathematische Beschreibung lassen sich diese Informationen nun in ein digitales Ökosystem einspeisen, zu dem auch das Streaming gehört, und sind so über alle möglichen Kanäle jederzeit verfügbar. Aber sie sind damit auch von der Geschichte ihrer Entstehung abgeschnitten. Das wird nun nochmals deutlicher, wenn wir keine DVDs mehr haben mit Kontextinformationen, wobei schon die meisten DVDs ihre Geschichte und ihre Quellen nicht dokumentieren.

Anders als die Utopie vermuten ließ, ist dieser universelle Zugang in der Praxis aber mit vielen Einschnitten verbunden, aus ökonomischen Gründen, die mit urheberechtlichen Fragen zusammenhängen. So sind manche Filme oder (Sprach-)fassungen nur an bestimmten Orten zugänglich, der Zugang wird durch Geoblocking kontrolliert. Oder es zirkulieren sehr fragwürdige, technisch und ästhetisch ungenügende Versionen auf offenen Kanälen wie YouTube.

3 Flückiger (2008).
AB: Welche neuen Forschungsfragen stellen sich durch das Sichten von Filmen im Video-Stream für die Filmwissenschaft und benachbarten Wissenschaften?

BF: Für die Forschung und Lehre ist Video-Streaming zunächst ein Segen, man muss nicht mehr DVDs oder BluRays bestellen, sondern kann unmittelbar auf die Filme zurückgreifen, was sich gerade während der Pandemie als sehr wichtig erwiesen hat. Denn so können wir online lehren und den Studierenden die Inhalte per Stream zur Verfügung stellen. Das ist ein Vorteil, solange wir die Kontrolle haben, welche Fassungen die Studierenden sehen, und diese Filme durch die Lehre entsprechend diskutieren können.

Ich würde weniger sagen, dass sich neue Forschungsfragen zum Gegenstand Film selbst stellen, aber neue Fragen zu den Feldern, die ich oben skizziert habe. Bis heute wird die Digitalisierung als viel zu transparent und neutral wahrgenommen, selbst in Fachkreisen, das müssen wir in der Filmwissenschaft stärker reflektieren und sowohl die materielle Grundlage wie auch die Genealogien der Digitalisierungen in den Blick nehmen.

Die Zirkulation und Rezeption ändert sich natürlich nachhaltig und das betrifft im Kontext der Arbeit mit Filmen vor allem auch das Dispositiv mit dem Direktzugriff. Wenn Studierende die Filme nicht mehr im Kollektiv visionieren, im abgedunkelten Seminarraum, sondern zu Hause oder irgendwo auf dem Laptop, Tablet oder vielleicht sogar Smartphone, und dann auch nicht mehr unbedingt am Stück oder ganz, sondern fragmentiert oder im Schnelldurchlauf, erleben sie Filme ganz anders. Das Dispositiv und die Rezeption sind daher wei- 


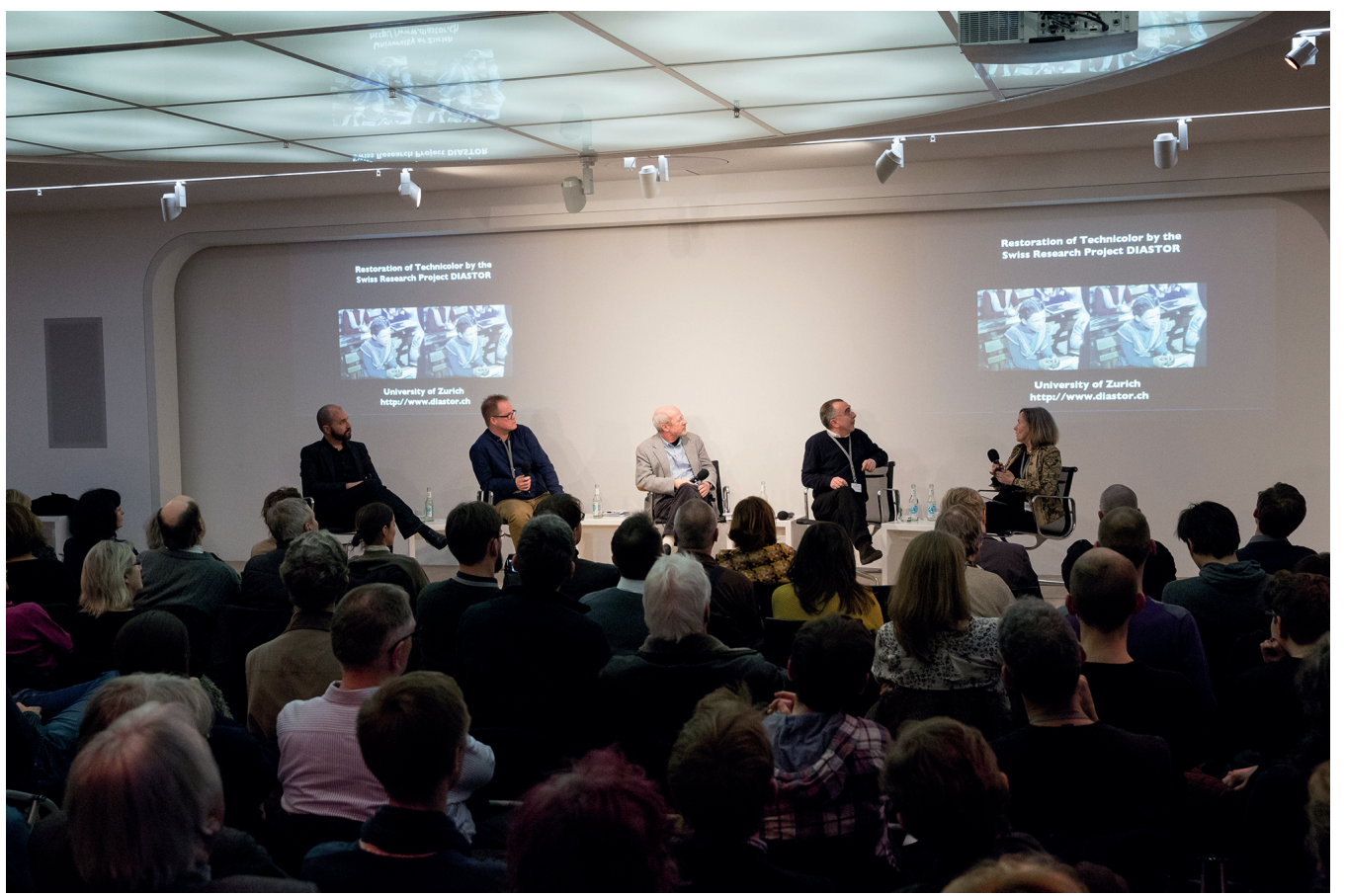

Abb. 2: DIASTOR: Präsentation von Resultaten an der Technicolor-Retrospektive, Berlinale $2015^{6}$ Foto: Sandra Weller

tere Aspekte, die wir in der Diskussion berücksichtigen müssen.

Mit der Digitalisierung eröffnen sich für die Forschung an Filmen als Werke neue Möglichkeiten durch digitale Werkzeuge wie Annotationssysteme oder Einbindungen in Plattformen über Semantic Web. Dazu werde ich unten genauer Stellung beziehen.

Ein Riesennachteil für Forschung und Lehre ist es aber, wenn die Filme als Dateien nicht verfügbar sind. Denn wir brauchen sie als Grundlage für Filmanalysen oder um Clips in unsere Vorlesungen und Seminare einzubinden. Das lässt sich über Streaming nur ungenügend bewältigen.

Frage AB: Das von Dir entwickelte Webportal zu historischen Farbfilmverfahren Timeline of Historical Filmcolors ${ }^{4}$ und das damit verbundene Forschungsprojekt wurde von der EU mit dem Advanced Grant ausgezeichnet und mit 2,9 Mio. Euro gefördert. Worauf zielt das Forschungsprojekt? Inwiefern sind die Forschungen zu historischen Filmfarben für die Digitalisierung von Filmen und den Zugang zu Filmen im Video-Stream relevant?

BF: Übergreifendes Ziel meiner Forschung zu Filmfarben ${ }^{5}$ ist es, den Zusammenhang zwischen technischen und

4 https://filmcolors.org/.

5 Siehe Flückiger (2020). ästhetischen Entwicklungen zu ergründen und systematisch darzustellen. Dazu gehört auch ganz besonders die materielle Ebene des Films, die wir einerseits über unsere fotografischen Dokumentationen in Filmarchiven untersuchen und auf der Timeline of Historical Film Colors publizieren, anderseits aber mit physikalisch-chemischen Methoden messen und auch diese Messungen auf der Timeline zur Verfügung stellen.

Schon in unserem Forschungsprojekt AFRESA ${ }^{7}$ zur Digitalisierung von Archivfilmen, das ab 2008 in Zusammenarbeit mit Rudolf Gschwind von der Universität Basel lief, habe ich festgestellt, dass die Übertragung von historischen Filmfarben in die digitale Domäne ein ungelöstes Problem darstellt. Die Vielfalt und die chemisch-physikalischen Eigenschaften sind sehr divergent. Aber professionelle Filmscanner sind nicht für diese Anforderungen entwickelt worden. Dazu haben wir in meinem Forschungsprojekt DIASTOR ${ }^{8}$ eine umfassende Scannerstudie durchgeführt, um die Defizite und Anforderungen systematisch zu erfassen und zu diskutieren, mit dem Ziel, ein Profil eines optimalen Scanners zu erstellen. Zuletzt habe ich ein Proof-of-Concept des European Research Council für die Entwicklung eines optimalen, multispek-

\footnotetext{
6 https://diastor.ch/results/.

7 https://www.aramis.admin.ch/Texte/?projectid=24948.

8 https://diastor.ch/.
} 

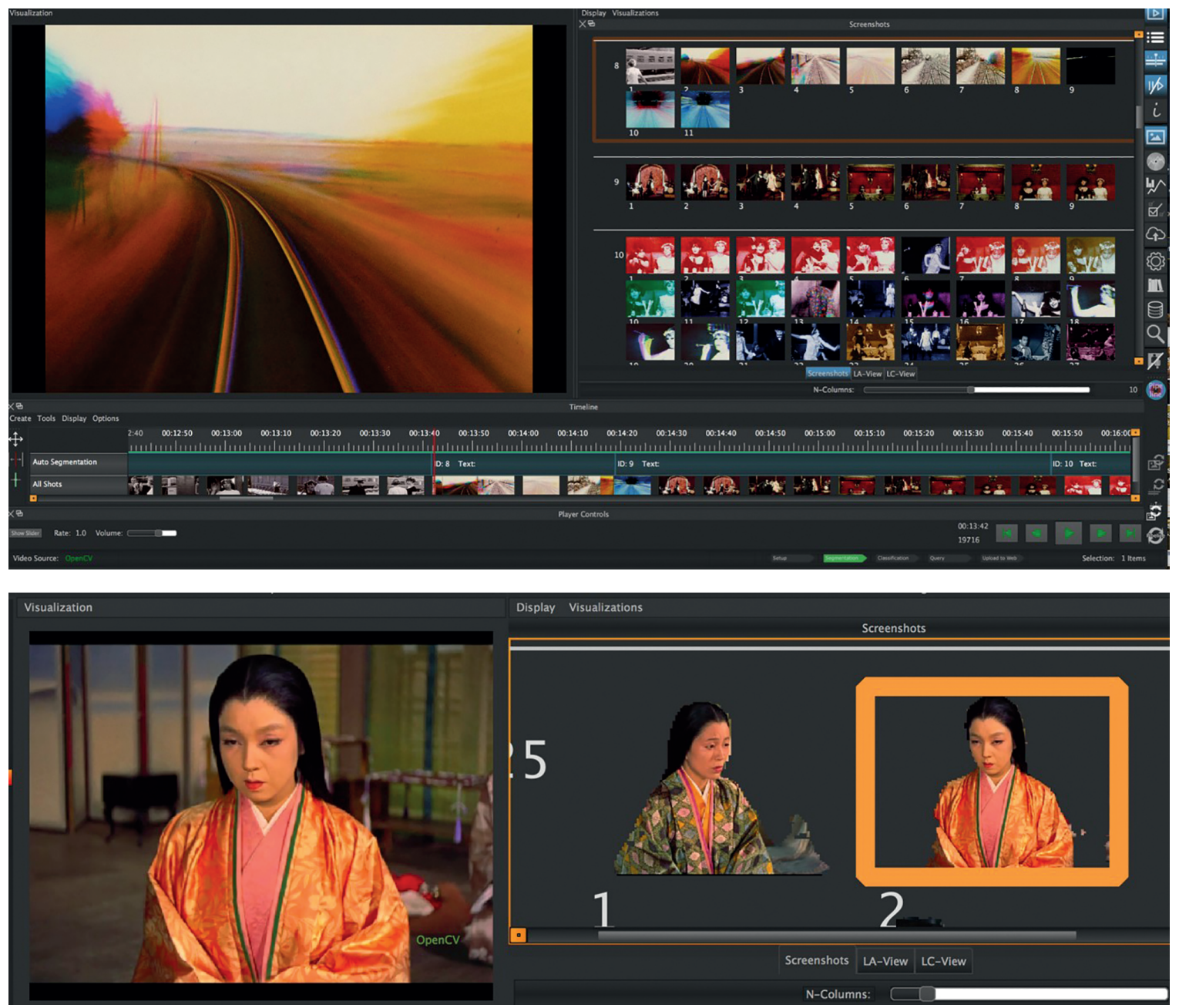

Abb. 3: VIAN Video-Annotations-Tool: Interface mit Segmentierungs-Tool und Screenshot-Manager (oben). Trennung von Figur und Grund mit Deep Learning Tools in VIAN (unten) ${ }^{10}$

tralen Scanners ${ }^{9}$ bekommen, und diesen Scanner haben wir als Prototyp auch gebaut ausgehend von einer Entwicklung von Lutz Garmsen, der auf mich zukam, und unter Einbezug der theoretischen Vorarbeiten, für die Giorgio Trumpy sein exzellentes Wissen eingebracht hat, und mit David Pfluger als Restaurierungsfachmann.

Diesen experimentellen Scanner haben wir mit einer exemplarischen Auswahl an historischen Filmmaterialien oder neu viragiertem und getontem Material mit historischen Rezepten durchgetestet, die Ulrich Rüdel von der HTW Berlin hergestellt hat. Die Resultate haben wir einer Auswahl von Fachexperten vorgestellt und mit ihnen evaluiert. Wir sind der Lösung schon sehr nahe. Nun habe ich als abschließendes Projekt noch einen Antrag beim

9 Blog-Posting von Claudy Op den Kamp: https://blog.filmcolors.or $\mathrm{g} / 2020 / 03 / 09 /$ vecoscan/.

10 Blog-Posting zu VIAN mit weiterführenden Links: https://blog.fil mcolors.org/2018/03/08/vian/.
Schweizerischen Nationalfonds gestellt, damit wir einen wissenschaftlich - filmhistorisch und physikalisch - wie auch restaurationsethisch wasserdichten Workflow entwickeln können, der zukunftsfähig ist.

Das zweite dominante Feld dieser Arbeit ist die Entwicklung von Analyseverfahren mit Methoden und Werkzeugen der Digital Humanities. Im ERC Advanced Grant FilmColors haben wir als Team - drei Doktorandinnen, drei Studierende, Bregt Lameris als Postdoc und ich - mehr als 400 Farbfilme vorwiegend aus der Zeit von 1895 bis 1995 mit einem computergestützten Ansatz analysiert. Der Analyseworkflow setzte sich zunächst aus einem Video-Annotations-Tool mit einem Netzwerk von Datenbanken zusammen. Ziel war es aber auch, spezifische Software für die Analyse zu entwickeln. Im Lauf der Zeit entstand ein ganzes Ökosystem von Werkzeugen, mit einem neuen VideoAnnotations-Tool namens VIAN für die visuelle Analyse, Annotation und Visualisierung einschließlich Kolorimetrie, entwickelt von Gaudenz Halter. Die Software verwen- 


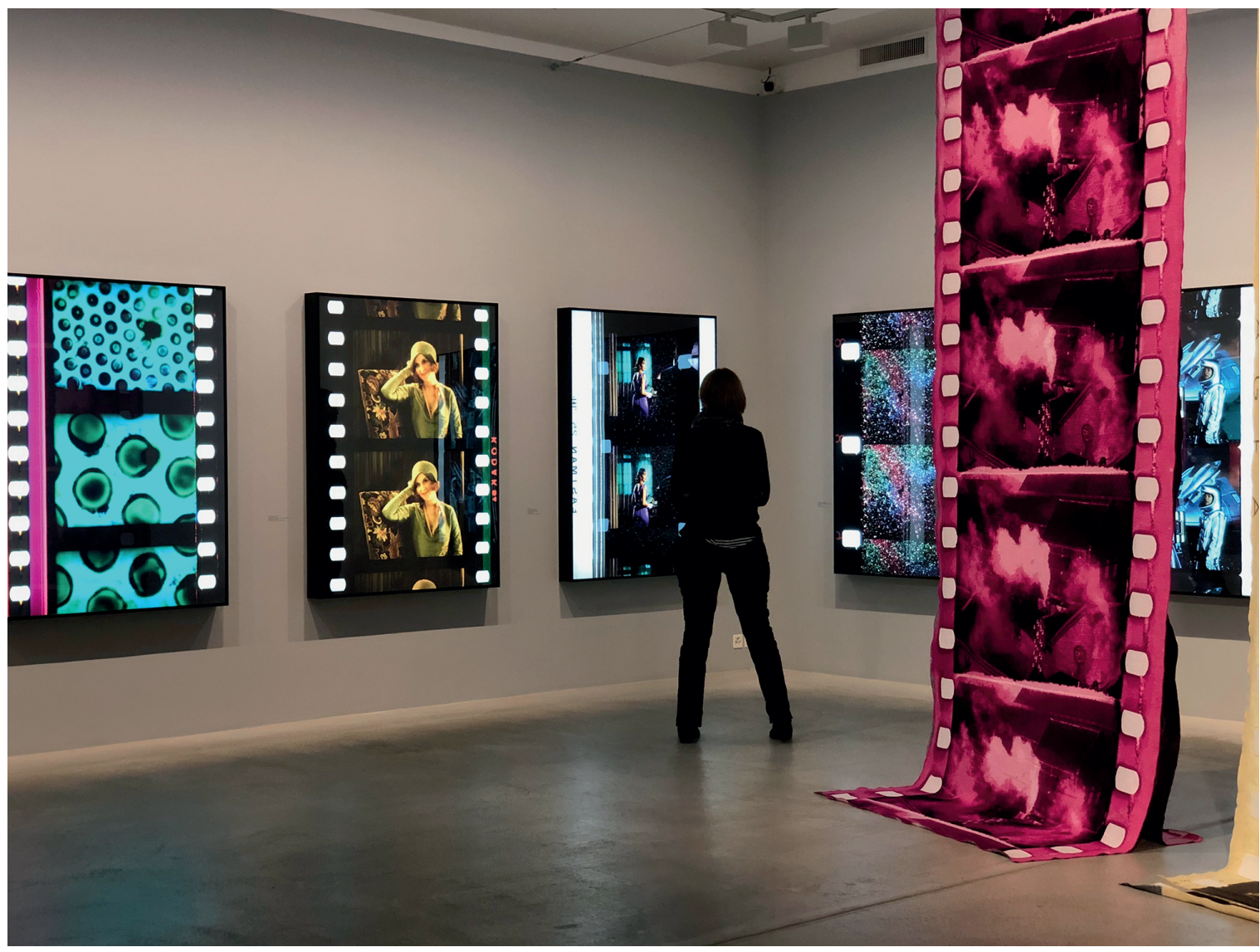

Abb. 4: Ausstellung Color Mania im Fotomuseum Winterthur ${ }^{13}$ Foto: Barbara Flückiger

det Ansätze des Deep Learning mit neuronalen Netzen, unter anderem um die Figuren vom Hintergrund zu lösen. Als zweites Modul des Ökosystems ist eine Crowd-Sourcing-Plattform entstanden, die VIAN WebApp, ${ }^{11}$ in der die gesamten Analysen für Abfragen und Visualisierungen auf Korpus-Ebene zugänglich sind und auf die in Zukunft auch externe Nutzer ihre Analysen aus VIAN hochladen können. ${ }^{12}$ Sie ist mit der Timeline of Historical Film Colors verbunden und auch mit einer App für unsere Ausstellung Color Mania im Fotomuseum Winterthur, welche den Besuchern Hintergrundinformationen zu den Exponaten lieferte.

AB: Welches Potential der Vernetzung von Informationen und Daten siehst Du zwischen Video-Streaming-Angeboten in Bibliotheken mit der filmwissenschaftlichen Forschung in den Digital Humanities? Welche Defizite

11 https://www.vian.app/static/manual/index.html, https://vimeo. com/396548709, https://vimeo.com/402360042, https://vimeo.com/ 404388151.

12 https://vimeo.com/user/70756694/folder/1220854.

$13 \mathrm{https} / /$ filmcolors.org/galleries/color-mania-exhibition/. oder Bedürfnisse gibt es aktuell beim Zugang zu Filmen per Video-Stream in der filmwissenschaftlichen Forschung und Lehre? Und was sollte eine Universitätsbibliothek leisten im Bereich Video-Streaming?

BF: Bibliotheken sind für mich ideale Partner, wenn es um Katalogisierung und Metadatendaten-Management, Ablage und Speicherung, Asset-Management und Verwaltung des Zugangs geht. Sie haben diese Kompetenz schon oder können sie aus ihren Kernkompetenzen entwickeln.

Wir stehen derzeit als Filmwissenschaft und als geisteswissenschaftliche Fakultät der Universität Zürich vor der Herausforderung, solche Strukturen zu schaffen oder eben mit großen Institutionen wie Bibliotheken Synergien zu entwickeln.

Ich stelle fest, dass es verschiedene einzelne Akteure gibt, die sehr visionär sind, aber als Ganzes haben weder die Geisteswissenschaften noch die Bibliotheken noch die Fördergremien wie zum Beispiel der Schweizerische Nationalfonds einen umfassenden Ansatz entwickelt, wie man langfristig mit diesen Ansätzen umgeht, wie man die verschiedenen Bausteine und Werkzeuge miteinander vernetzt und die Nachhaltigkeit sicherstellt. 
Am einfachsten ist es immer, Hardware zu kaufen. Aber die Datenpflege, die konzeptuelle Entwicklung, die fortlaufende Anpassung von Softwares und Repositorien sind arbeitsintensiv und bedürfen spezieller Kompetenz von Fachexperten mit einem möglichst interdisziplinären Profil, welche die Seite der IT verstehen, aber auch die theoretische Reflexion und wissenschaftliche Kontextualisierung der Ansätze, Methoden und Werkzeuge. Derzeit ist dies alles $\mathrm{zu}$ wenig systematisiert aufgegleist, sondern hängt von Einzelinitiativen und Projekten ab. So sind viele Digital-Humanities-Werkzeuge wieder verschwunden, weil sie nach Abschluss von Projekten nicht weiter gepflegt wurden.

Wir haben Leuchtturm-Projekte wie CLARIAH (Common Lab Research Infrastructure for the Arts and Humanities $)^{14}$ in den Niederlanden, die eine umfassende Infrastruktur für die Geisteswissenschaften aufbauen, inklusive einer Media Suite für die Bearbeitung von audiovisuellen Daten. Wir haben in den USA das Media Ecology Project ${ }^{15}$ von Mark Williams oder die Media History Digital Library ${ }^{16}$ und das Project Lantern ${ }^{17}$ zur Suche und Visualisierung von schriftlichen Quellen.

Aber wir haben zu wenig Standardisierung und Kooperation zwischen den verschiedenen Ansätzen. Das ist meine nächste Mission: Dass ich mehr Zusammenarbeit der verschiedenen Akteure anstrebe, mit dem Ziel, die einzelnen Lösungen über offene Standards miteinander zu verknüpfen und eine Superstruktur aufzubauen, in der alle Ansätze - auch neue - andocken können.

\section{Literaturverzeichnis}

Flückiger, Barbara (2008): Visual Effects. Filmbilder aus dem Computer. Marburg: Schüren.

Flückiger, Barbara (2020): Filmfarben. Materialität, Technik, Ästhetik. In: Color Mania. Materialität Farbe in Fotografie und Film, hg. v. Barbara Flückiger, Eva Hielscher and Nadine Wietlisbach. Zürich, Winterthur: Lars Müller. Fotomuseum Winterthur, 17-49.

\section{Prof. Dr. Barbara Flückiger}

Seminar für Filmwissenschaft der Universität Zürich

Projektleiterin und Principal Investigator des Projekts ERC

Advanced Grant FilmColors. Bridging the Gap Between Technology

and Aesthetics

Affolternstrasse 56

$\mathrm{CH}-8050$ Zürich

Schweiz

baflueckiger@gmail.com

https://orcid.org/0000-0003-4954-5469

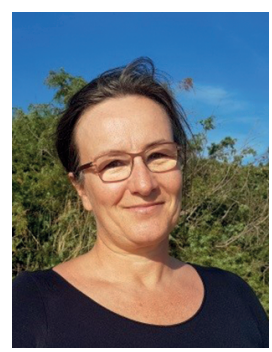

\section{Dr. Anna Bohn}

Zentral- und Landesbibliothek Berlin

Öffentliche Zentralbibliothek

Wissenschaftliche Referentin Stabsstelle Strategische Filmentwicklung

Breite Str. 30-36

10178 Berlin

anna.bohn@zlb.de

https://orcid.org/0000-0001-9625-3069

AB: Herzlichen Dank für das Interview.

14 https://www.clariah.nl/.

$15 \mathrm{http}: / /$ mediaecology.dartmouth.edu/wp/.

16 https://mediahistoryproject.org/.

17 https://lantern.mediahist.org/. 